\title{
Comment se forment les nuages ? L'expérience du nuage dans une bouteille
}

\author{
Camille Risi, Jean-Louis Dufresne, Jean-Yves Grandpeix, \\ Sonia Labetoulle, Geneviève Sèze, Aymeric Spiga \\ Laboratoire de météorologie dynamique, Institut Pierre-Simon Laplace, CNRS, \\ Université Pierre-et-Marie-Curie, 4 place Jussieu, 75252 Paris Cedex 05 \\ camille.risi@Imd.jussieu.fr
}

e but de cette expérience est d'expliquer comment se créent les nuages dans l'atmosphère en formant artificiellement un «nuage » dans une bouteille. Cette expérience peut convenir au grand public et aux élèves des écoles primaires, qui apprécient son côté spectaculaire, même s'ils ne mâ̂trisent pas toutes les notions physiques sous-jacentes. Elle peut aussi convenir aux collégiens, aux lycéens et aux étudiants du supérieur, en classe ou dans le cadre de travaux personnels encadrés (TPE) ou de travaux d'initiative personnelle encadrés (TIPE). L'exploitation pédagogique est en effet très adaptable au niveau du public.

\section{Montage et déroulement de l'expérience}

Plusieurs montages de bouteilles existent et nous en présentons deux ici (figure 1). Le premier est le plus facile

But pédagogique : mettre en évidence comment se forment les nuages

Public : du CE2 à l'enseignement supérieur, discours adaptable

Matériel nécessaire : deux bouteilles transparentes en plastique ou une bouteille en verre avec un tensiomètre médical ; de la colle ; un poinçon pour percer le bouchon ; une source de lumière (lampe torche LED, projecteur de diapositives, vidéoprojecteur, ou laser); un peu d'eau; des allumettes.

\section{Site internet :}

http://www.ipsl.fr/fr/Pour-tous/Espacepedagogique/Faire-un-nuage-dans-unebouteille

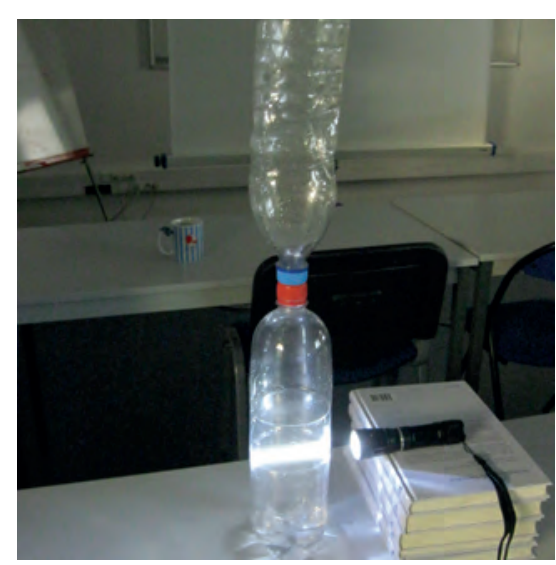

à réaliser, le second le plus simple à utiliser.

1) Montage artisanal : deux bouteilles transparentes en plastique sont assemblées hermétiquement au niveau de leurs bouchons respectifs. Les bouchons sont percés d'un trou, pour permettre le passage de l'air entre les bouteilles, puis collés ensemble. La bouteille inférieure est celle où on visualise le nuage ; la bouteille supérieure sert, par écrasement, à provoquer la compression et, par relâchement, la détente nécessaire à la formation du nuage.

2) Montage avec tensiomètre médical : un tensiomètre médical est fixé sur le bouchon d'une bouteille transparente en verre via un tuyau flexible. La poire du tensiomètre sert à faire monter la pression, et la soupape à provoquer la détente. Un montage du même type est possible avec une pompe à vélo et une valve montée sur le bouchon, mais celle que nous proposons ici s'est révélée non seulement plus facile d'usage mais aussi plus fiable.

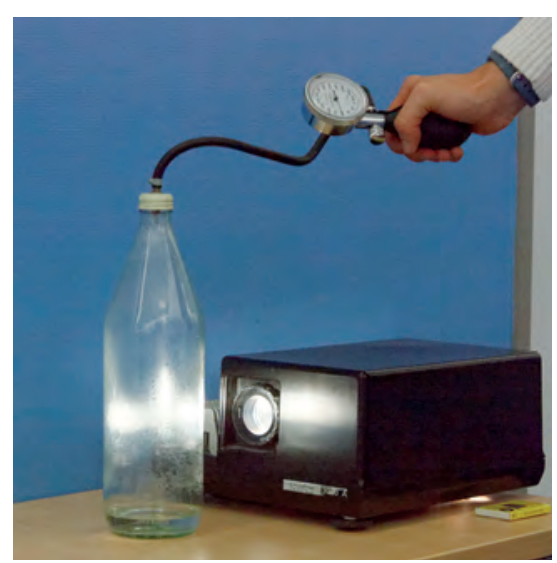

Figure 1. Montage $n^{\circ} 1$ : deux bouteilles transparentes en plastique sont reliées entre elles par deux bouchons percés et collés entre eux (à gauche). Montage $n^{\circ} 2$ : une bouteille en verre et son bouchon équipé d'un manomètre, d'une soupape et d'une poire provenant d'un tensiomètre médical (à droite). 
Une description plus détaillée de ces montages est accessible sur le site internet indiqué plus haut.

Pour visualiser le nuage, la bouteille doit être illuminée par un faisceau de lumière. Celui-ci peut provenir d'un projecteur de diapositives, d'un vidéoprojecteur ou d'une lampe torche à LED assez puissante. Il faut que l'expérience se tienne dans un lieu sans lumière directe du soleil, et si possible pas trop éclairé. Le nuage se verra mieux en regardant dans une direction qui fait un angle entre 30 et $150^{\circ}$ avec le faisceau de lumière, et il ne faut pas hésiter à essayer plusieurs angles de vue (figure 1).

Pour créer le nuage, il faut :

- mettre un fond d'eau dans la bouteille à nuage, et ce suffisamment en avance pour que l'ensemble soit à température ambiante et uniforme ;

- augmenter la pression dans la bouteille, en appuyant sur la bouteille du haut pour le montage artisanal, ou en pompant avec la poire pour le montage avec le tensiomètre médical ;

- agiter et attendre un peu pour que le dispositif revienne en équilibre thermique avec l'extérieur et pour supprimer la buée qui s'est éventuellement formée sur les bords internes de la bouteille;

- diminuer brusquement la pression en relâchant la bouteille du haut ou en dévissant la vis de la soupape, selon le montage.

Le nuage se forme : des gouttelettes en suspension tourbillonnent, puis retombent lentement sous forme d'une bruine sous l'effet de la gravité. L'expérience peut être renouvelée autant de fois que souhaité.

Pour rendre le nuage formé plus visible, on pourra ajouter un peu de fumée (voir ci-dessous).

\section{Exploitation pédagogique}

L'exploitation de cette expérience dépend du niveau du public. Avant tout, il convient de rappeler qu'un nuage est formé de gouttelettes d'eau ou de cristaux de glace en suspension dans l'atmosphère. La vapeur d'eau, quant à elle, est invisible.

Pourquoi des gouttelettes se formentelles lorsque l'on diminue la pression dans la bouteille?

L'explication peut être décomposée en deux étapes. Premièrement, lorsque la pression diminue, la température diminue. Deuxièmement, lorsque la température diminue, la vapeur d'eau présente dans l'air se condense sous forme de gouttelettes qui elles sont visibles.

\section{Précisons ces deux étapes.}

Première étape : la température d'un gaz peut diminuer lorsqu'on lui soustrait de l'énergie ; une façon de le faire est de baisser sa pression. C'est ce qui se passe lorsque l'on relâche la bouteille du haut ou que l'on dévisse la soupape du tensiomètre, à la manière d'une bombe à crème Chantilly qui se refroidit lorsque l'on éjecte une partie du gaz propulseur qu'elle contient. Inversement, on peut observer un échauffement de l'air contenu dans un pneu de vélo quand il est gonflé avec une pompe. Pour les petits, on peut expliquer avec les mains le lien entre pression et température. Pour les grands, cela s'appelle une détente adiabatique qui est étudiée en thermodynamique.

L'énergie apportée par la compression a le temps de se dissiper et la température de l'air dans la bouteille retourne à sa valeur initiale. La décompression, au contraire, est effectuée rapidement pour que la température de l'air diminue significativement.

Deuxième étape : à température ambiante, l'eau peut être sous forme liquide (des gouttelettes dans le cas d'un nuage) ou sous forme gazeuse (de la vapeur d'eau invisible). La quantité d'eau maximale qui peut exister sous forme gazeuse dépend de la température de l'air, selon une relation dite de Clausius-Clapeyron qui sera détaillée dans un prochain article.

Lorsque cette valeur maximale est atteinte, on dit que l'on est à la saturation. À ce stade, une simple baisse de la température entraîne la condensation d'une partie de la vapeur d'eau sous forme de gouttelettes. C'est ce qui se passe lorsque l'on baisse la pression, et donc la température, dans la bouteille.

\section{Et dans la nature, comment se forment les nuages?}

Prenons l'exemple des nuages de beau temps que l'on appelle aussi des cumulus (figure 2, gauche). Lorsqu'il fait beau, le sol se réchauffe sous l'effet des rayons du soleil et atteint sa température maximale dans l'après-midi. L'air réchauffé par la surface devient moins dense et donc s'élève. Lors de l'ascendance de l'air, sa pression diminue (car la pression, à une altitude donnée, est associée au poids de la colonne d'air au-dessus de cette altitude). La diminution de pression entraîne une diminution de température et donc la condensation de la vapeur d'eau. Le nuage se forme ainsi au cours de l'ascendance de l'air. C'est
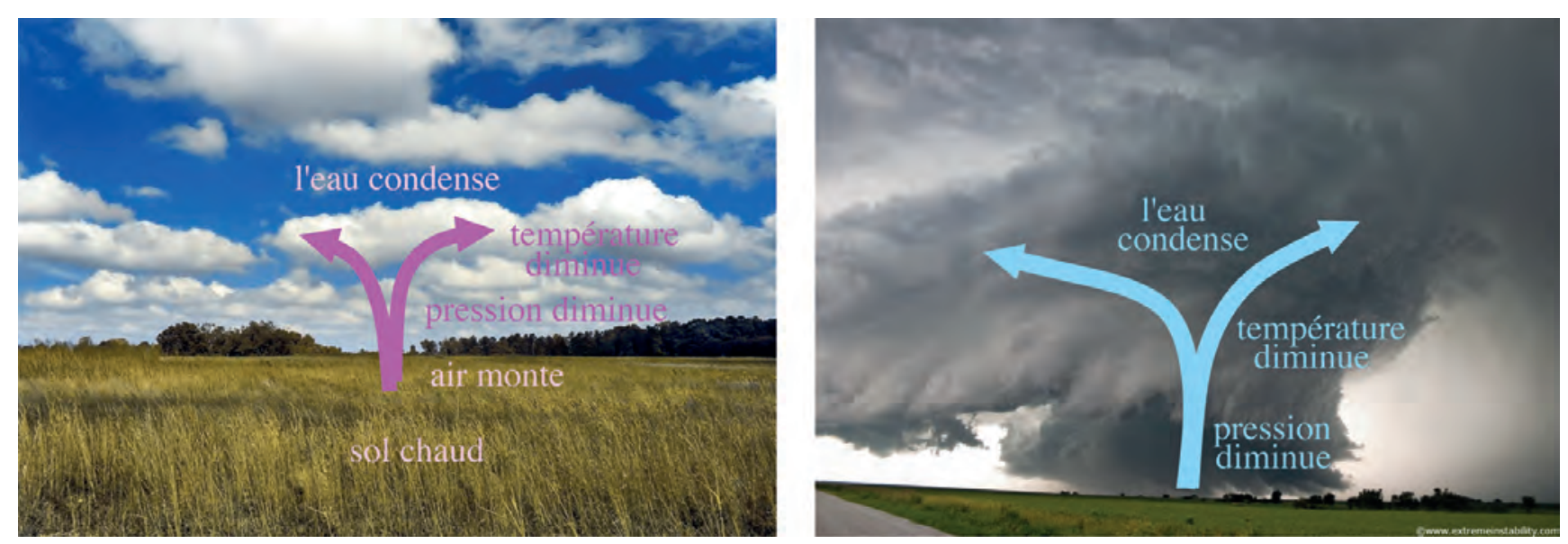

Figure 2. À gauche : formation d'un cumulus. À droite : formation d'un cumulonimbus. 
pourquoi il y a souvent des cumulus les après-midis des jours de beau temps en été. Si l'atmosphère est particulièrement instable ce jour-là, l'air monte beaucoup plus haut, jusqu'à 10-15 km d'altitude dans les tropiques, et il se forme alors un nuage sur toute l'ascendance, que l'on appelle cumulonimbus (figure 2, droite). C'est un nuage d'orage, qui se développe souvent en fin d'après-midi l'été.

\section{Effet des aérosols}

L'expérience de formation des nuages peut aussi permettre d'aborder le rôle des aérosols (petites particules en suspension dans l'atmosphère que l'on appelle parfois poussières) sur les propriétés des nuages. Pour ajouter des aérosols dans la bouteille, il suffit d'y faire entrer un peu de fumée : on peut utiliser pour cela une allumette ou un bâton d'encens. Pour "nettoyer » l'air des aérosols, il suffit de répéter l'expérience de formation des nuages plusieurs fois, de façon à ce que les gouttelettes piègent les aérosols et finissent par les mélanger à l'eau liquide au fond de la bouteille.

Quand la concentration en aérosols est faible (comme c'est le cas dans l'air ambiant), les gouttelettes sont suffisamment grosses pour être visibles individuellement à l'œil nu (figure 3, gauche). Elles retombent alors assez rapidement sous l'action de leur propre poids. Au contraire, quand la concentration en aérosols est élevée, les gouttelettes sont beaucoup plus fines, voire microscopiques. Le nuage dans son ensemble est beaucoup plus brillant. On voit se former de fins filaments d'air plus ou moins nuageux qui matérialisent les mouvements de convection et de turbulence dans la bouteille (figure 3, droite).

Cette différence s'explique par le fait que les gouttelettes se forment autour des noyaux de condensation que sont les aérosols. Ainsi, plus il y a d'aérosols, plus nombreuses sont les gouttelettes. Â quantité d'eau condensée égale, ce qui est le cas ici puisque celle-ci ne dépend que du refroidissement induit par la détente adiabatique, des gouttelettes plus nombreuses sont plus petites. La surface collective des gouttelettes qui diffusent la lumière est donc plus élevée et le nuage apparaît plus brillant.

Dans la nature, les aérosols naturels ou émis par les activités humaines agissent de la même manière sur les
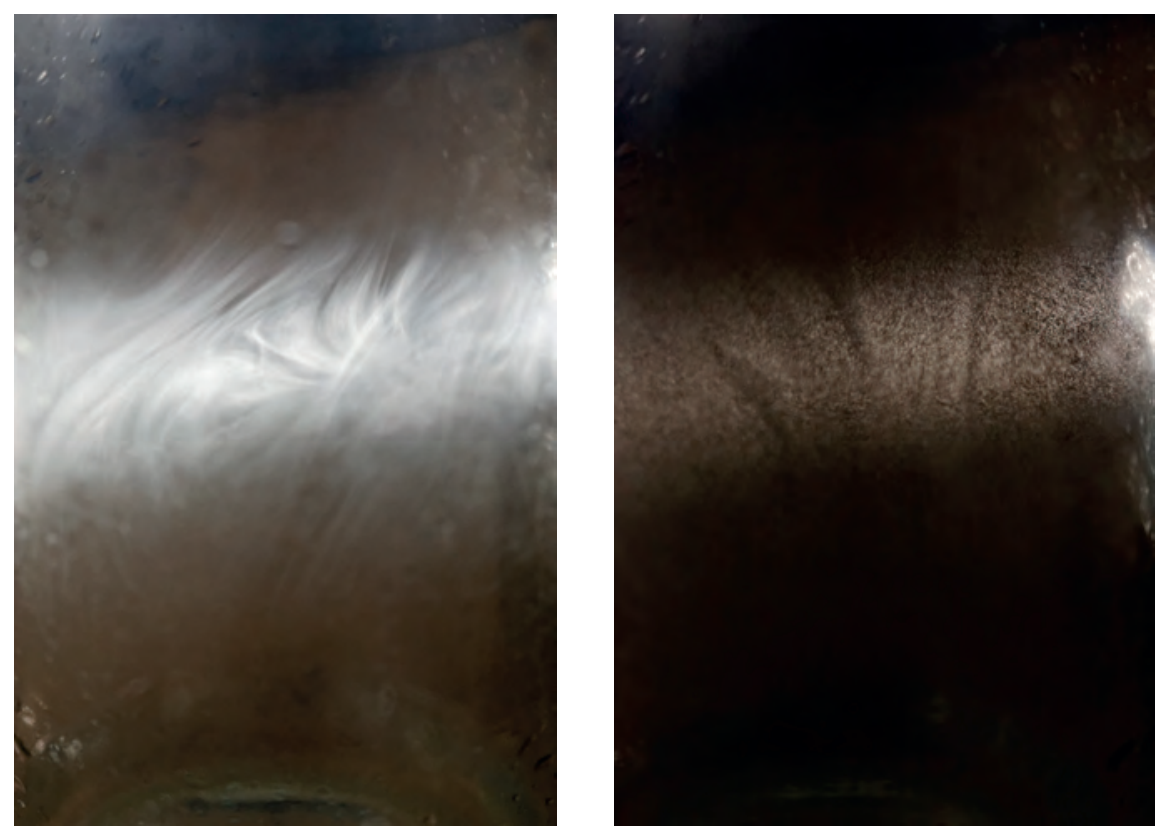

Figure 3. Les deux types de nuages que l'on peut former en fonction du contenu de l'air en aérosols. Les deux photos ont été prises avec la même résolution. A gauche, lorsque l'air de la bouteille est propre, les gouttelettes sont visibles à l'œil nu. À droite, lorsqu'on introduit des aérosols dans la bouteille, les gouttelettes qui se forment sont beaucoup plus petites. Le nuage est plus brillant, plus opaque et on voit des volutes, qui matérialisant les mouvements de convection et de turbulence dans la bouteille. propriétés nuageuses. Des concentrations importantes en aérosols conduisent à la formation de nuages plus réfléchissants, qui contribuent à refroidir la Terre (Boucher, 1997). Toutefois, les interactions entre nuages et aérosols sont complexes et le rôle des aérosols sur le climat est encore mal compris. Il n'en reste pas moins qu'au premier ordre la formation et les propriétés des nuages dépendent avant tout de la circulation atmosphérique : ascendance de l'air, mélange de masses d'air, refroidissement radiatif, etc.

\section{Dans la vie courante}

Dans le langage courant, on appelle parfois à tort « vapeur » les gouttelettes d'eau qui se forment dans l'air. C'est ainsi qu'on parle de «vapeur » au-dessus d'une casserole, alors que ce sont en fait des gouttelettes d'eau que l'on voit. Par ailleurs, il n'est pas connu de tous que l'air ambiant contient de la vapeur d'eau, mais une expérience très simple permet de le prouver. Si l'on prend une bouteille d'eau sortant du réfrigérateur, on voit apparaître très rapidement de l'eau liquide sur la bouteille. L'air se refroidit au contact de la bouteille ; la vapeur sur la surface de celle-ci. La buée que l'on peut observer sur les vitres les jours où il fait froid répond au même phénomène. La vapeur d'eau est en fait toujours présente dans l'air, mais à des concentrations très variables. Il suffit de refroidir suffisamment cet air pour que l'eau se condense.

\section{Conclusion}

Cette expérience permet d'aborder la formation des nuages de manière très simple. Elle peut toutefois être étendue dans le cadre de TPE ou TIPE pour étudier les processus microphysiques de manière plus complexe, pour quantifier l'effet des aérosols sur les propriétés radiatives des nuages formés dans la bouteille ou bien d'autres choses encore. Le TIPE de Romain Faucher en est un très bon exemple. d'eau ambiante peut alors se condenser

\section{Bibliographie}

Boucher 0., 1997. L'influence climatique des aérosols, La Météorologie, 17, 11-22.

Faucher R., 2006. Rapport de stage, Expériences sur la formation des nuages.

http://www.Imd.jussieu.fr/ jldufres/Manip/Formationdesnuages5.pdf 


\section{ANNONCE CONCOURS ÉTABLISSEMENTS SCOLAIRES}

Fondée en 1852, reconnue d'utilité publique.

Agréée par le ministere de l'Education nationaie.

\section{PRIX PERRIN DE BRICHAMBAUT 2015}

\section{Un concours national " météo et climat " pour les écoles, les collèges et les lycées}
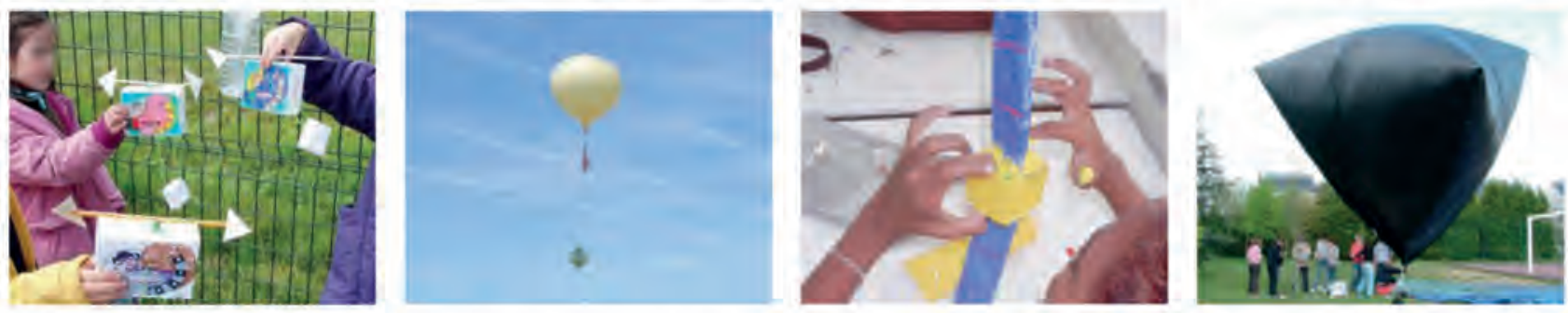

Ce prix a été créé en 1997 par la SMF-Météo et Climat afin d'honorer la mémoire de Christian Perrin de Brichambaut (1928-1995), ancien ingénieur à Météo-France et ancien président de la SMF-Météo et Climat qui a beaucoup œuvré en faveur de la vulgarisation scientifique.

\section{QUI PEUT CONCOURIR?}

Ce concours est réservé aux écoles primaires, aux collèges et aux lycées qui réalisent un projet sur le thème de la météorologie ou du climat au cours de l'année scolaire 2014-2015.

Les établissements scolaires étrangers sont autorisés à concourir dans la mesure où le projet soumis et ses annexes sont rédigés en langue française.

\section{JURY}

Les projets sont évalués par un jury composé de météorologistes et d'enseignants. La démarche scientifique, l'implication des élèves et la présentation des projets sont des critères majeurs d'appréciation.

\section{DOTATIONS}

$\begin{array}{ll}\text { 1er prix : }^{\text {e }} & 700 € \\ \text { 2 }^{\mathrm{e}} \text { prix: } & 500 € \\ \text { 3 }^{\mathrm{p}} \text { prix : } & 300 €\end{array}$

\section{COMMENT CONCOURIR AU PRIX 2015 ?}

II suffit d'envoyer un dossier comprenant :

$\checkmark$ Une lettre de candidature avec coordonnées postales de l'établissement, adresse e-mail et numéro de téléphone de l'enseignant responsable du projet, niveau de la classe participant au concours.

$\checkmark$ Le descriptif du projet (une page A4 maximum).

$\checkmark$ Tous les supports permettant au jury d'apprécier le projet : dossier de présentation illustré, DVD, vidéos...

\section{OÙ ENVOYER LE DOSSIER?}

SMF-Météo et Climat

73, avenue de Paris, 94165 Saint-Mandé Cedex ou par e-mail : morgane.daudier@meteoetclimat.fr

\section{EXEMPLES DE PROJETS}

Lâchers de ballons sondes, observations et mesures en météorologie, création d'un site Internet, construction d'instruments...

\section{DATE LIMITE DE LA RÉCEPTION DU DOSSIER 31 mai 2015 \\ Date de réception de votre candidature dans nos bureaux de Saint-Mandé. Veuillez tenir compte des délais postaux.}

\section{ATTRIBUTION DES PRIX}

La décision d'attribution des prix sera communiquée avant la fin de l'année scolaire et le $1^{\text {er }}$ prix sera remis dans l'établissement en juin 2015.
Concours organisé par l'association SMF-Météo et Climat

www.meteoetclimat.fr

\section{Avec le soutien de}

\section{METEO FRANCE}


